\title{
Two-Dimensional Inorganic Cationic Network of Thorium Iodate Chloride with Unique Halogen-Halogen Bonds
}

Huangjie $\mathrm{Lu}^{1,2 \ddagger}$, Yaxing Wang ${ }^{1,2 \ddagger}$, Congzhi Wang ${ }^{\star 3}$, Lanhua Chen ${ }^{1,2}$, Weiqun Shi, ${ }^{3}$ Juan Diwu ${ }^{1,2}$, Zhifang Chai ${ }^{1,2}$, Thomas E. Albrecht-Schmitt ${ }^{4}$, and Shuao Wang*1,2

${ }^{1}$ School for Radiological and interdisciplinary Sciences (RAD-X), Soochow University,199 Ren'ai Road,Suzhou 215123, China

${ }^{2}$ Collaborative Innovation Center of Radiation Medicine of Jiangsu Higher Education Institutions, 199 Ren'ai Road, Suzhou 215123, China

${ }^{3}$ Laboratory of Nuclear Energy Chemistry and Key Laboratory for Biomedical Effects of Nanomaterials and Nanosafety, Institute of High Energy Physics, Chinese Academy of Sciences, Beijing 100049, China

\$These authors contributed equally. 
Table of contents

\section{S1. EDS Data}

S2. Powder X-ray diffraction (PXRD)

S3. Anion-exchange test

S4. Selected bond lengths and angles

S5. Quantum chemical calculations 
S1. EDS Data. Scanning electron microscopy images and energy-dispersive spectroscopy data were recorded on a FEI Quanta 200FEG Scanning Electron Microscope with the energy of the electron beam being $30 \mathrm{keV}$. Samples were mounted directly on the carbon conductive tape with Au coating.

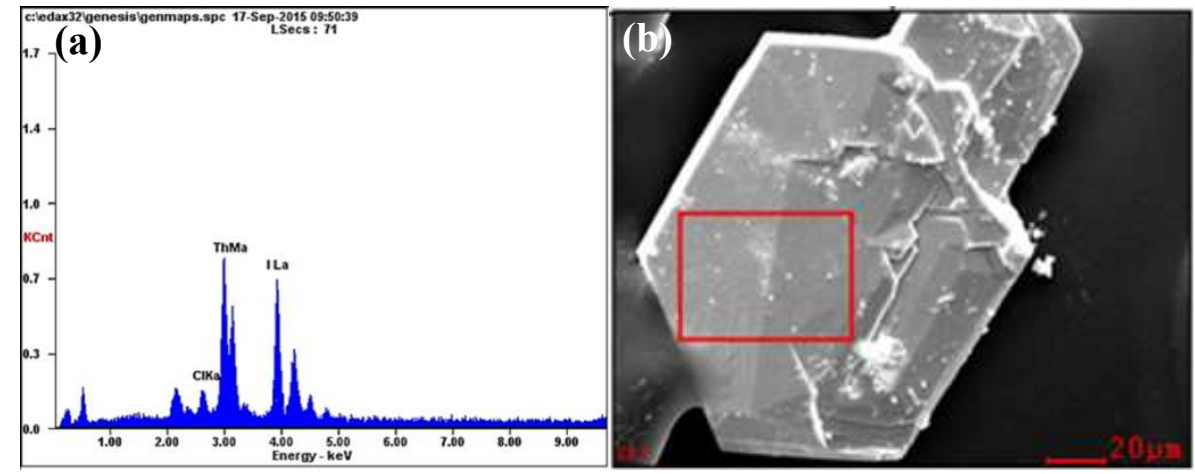

Figure S1. EDS (a) and SEM (b) images of compound ThIOCl.

Table S1. EDS data of compound ThIOCl.

\begin{tabular}{|c|c|c|}
\hline Element & $\boldsymbol{W t} \%$ & $\boldsymbol{A t} \%$ \\
\hline $\boldsymbol{O K}$ & 10.33 & 51.34 \\
\hline $\boldsymbol{C l K}$ & 02.88 & 06.47 \\
\hline $\boldsymbol{T h} \boldsymbol{M}$ & 42.88 & 15.31 \\
\hline $\boldsymbol{I L}$ & 42.90 & 26.87 \\
\hline Matrix & Correction & ZAF \\
\hline \hline
\end{tabular}

S2. Powder X-ray diffraction (PXRD). The experimental PXRD parttern of ThIOCl reveals that it is a single phase without any impurities.

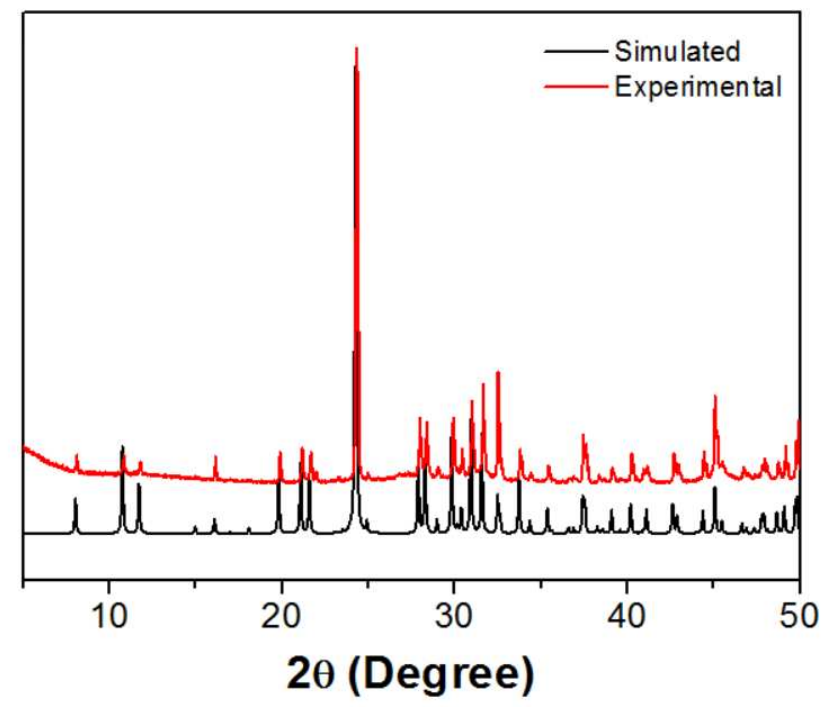


Figure S2. Simulated and experimental PXRD patterns for compound ThIOCI confirming the phase-purity.

\section{S3. Anion-exchange test:}

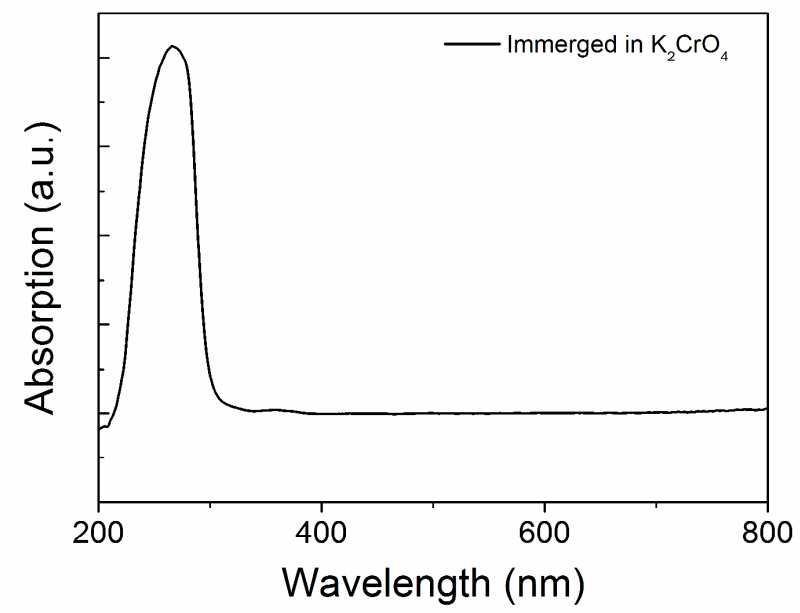

Figure S3. UV-Vis spectrum of compound ThIOCl immerged in $\mathrm{K}_{2} \mathrm{CrO}_{4}$ for $12 \mathrm{~h}$ at room temperature.

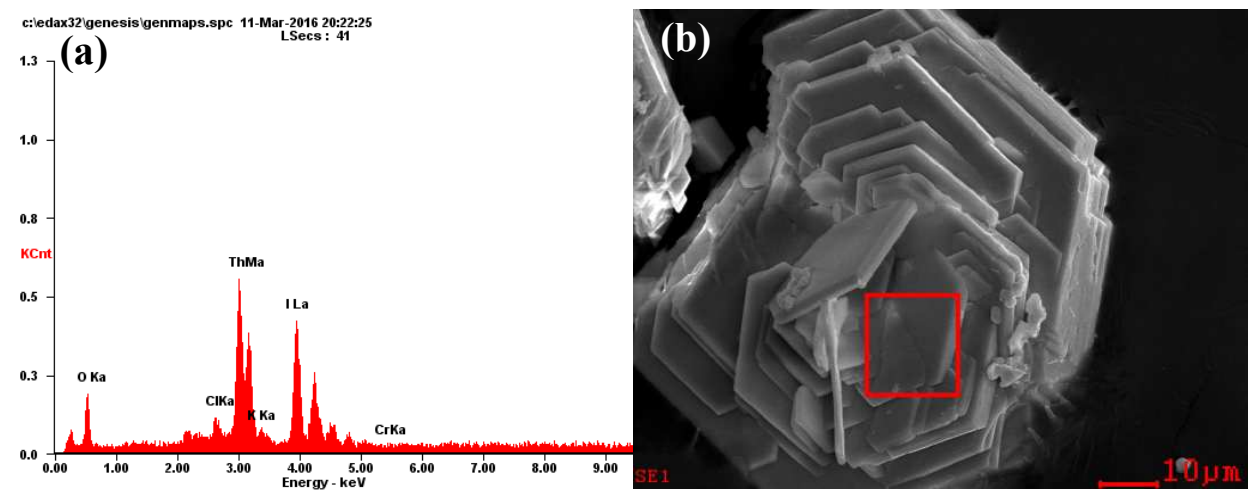

Figure S4. EDS (a) and SEM (b) images of ThIOCl soaked in $0.1 \mathrm{M} \mathrm{K}_{2} \mathrm{CrO}_{4}$ solution for $12 \mathrm{~h}$ at room temperature. 
Table S2. EDS data of compound ThIOCI soaked in $0.1 \mathrm{M} \mathrm{K}_{2} \mathrm{CrO}_{4}$ solution for $12 \mathrm{~h}$ at room temperature.

\begin{tabular}{|c|c|c|}
\hline Element & $\boldsymbol{W t} \%$ & $\boldsymbol{A t} \%$ \\
\hline $\boldsymbol{O K}$ & 18.93 & 68.16 \\
\hline $\boldsymbol{C l K}$ & 02.34 & 03.80 \\
\hline $\boldsymbol{T h M}$ & 41.44 & 10.29 \\
\hline $\boldsymbol{K} \boldsymbol{K}$ & 00.68 & 01.00 \\
\hline $\boldsymbol{I L}$ & 36.43 & 16.54 \\
\hline CrK & 00.18 & 00.20 \\
\hline Matrix & Correction & ZAF \\
\hline \hline
\end{tabular}

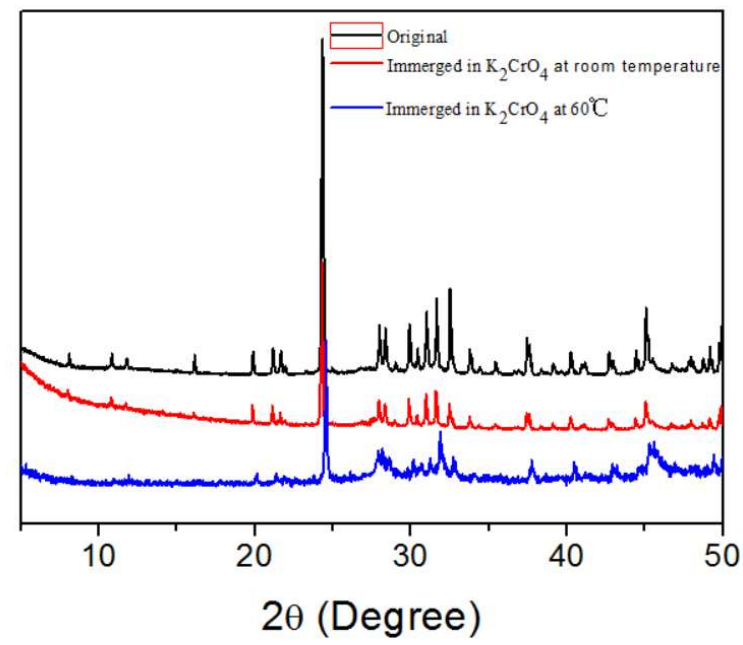

Figure S5. Experimental PXRD patterns for original compound ThIOCl and ThIOCl immerged in $0.1 \mathrm{M} \mathrm{K}_{2} \mathrm{CrO}_{4}$ solution at room temperature and $60{ }^{\circ} \mathrm{C}$.

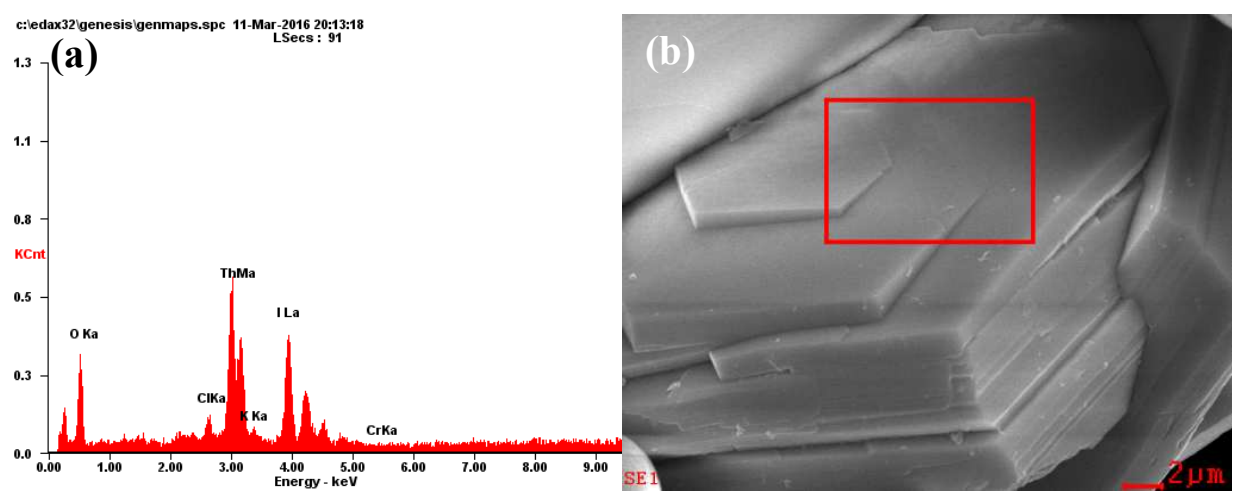


Figure S6. EDS (a) and SEM (b) images of compound ThIOCl soaked in $0.1 \mathrm{M} \mathrm{K}_{2} \mathrm{CrO}_{4}$ solution for $12 \mathrm{~h}$ at $60^{\circ} \mathrm{C}$.

Table S3. EDS data of compound ThIOCl soaked in $0.1 \mathrm{M} \mathrm{K}_{2} \mathrm{CrO}_{4}$ solution for $12 \mathrm{~h}$ at $60^{\circ} \mathrm{C}$.

\begin{tabular}{|c|c|c|}
\hline Element & Wt\% & At\% \\
\hline OK & 26.85 & 76.93 \\
\hline CIK & 02.19 & 02.83 \\
\hline ThM & 37.24 & 07.36 \\
\hline KK & 00.76 & 00.89 \\
\hline IL & 32.79 & 11.84 \\
\hline CrK & 00.18 & 00.16 \\
\hline Matrix & Correction & ZAF \\
\hline
\end{tabular}

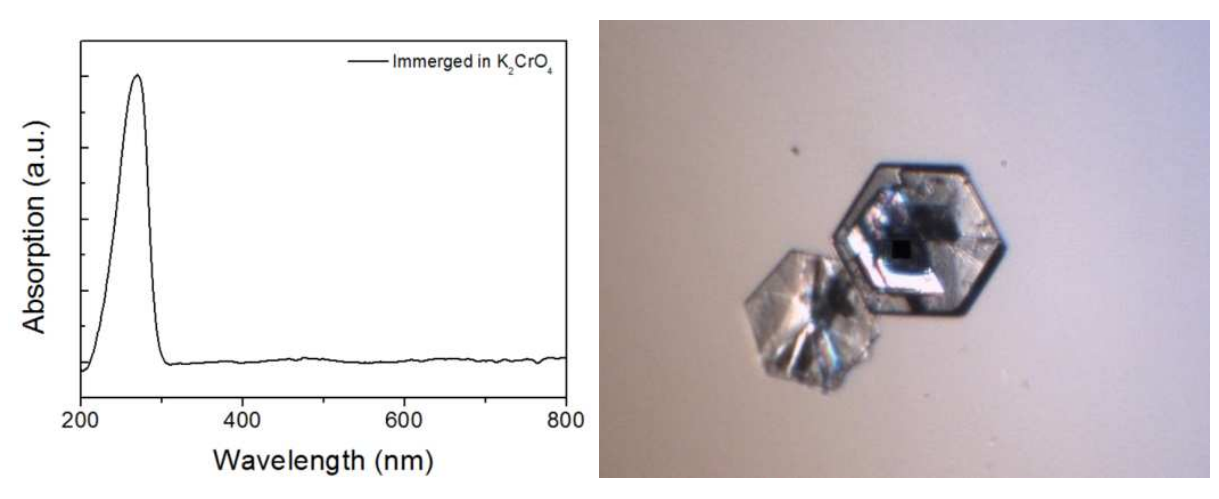

Figure S7. UV-Vis spectrum of compound ThIOCl soaked in $0.1 \mathrm{M} \mathrm{K}_{2} \mathrm{CrO}_{4}$ solution for $12 \mathrm{~h}$ at $60^{\circ} \mathrm{C}$.

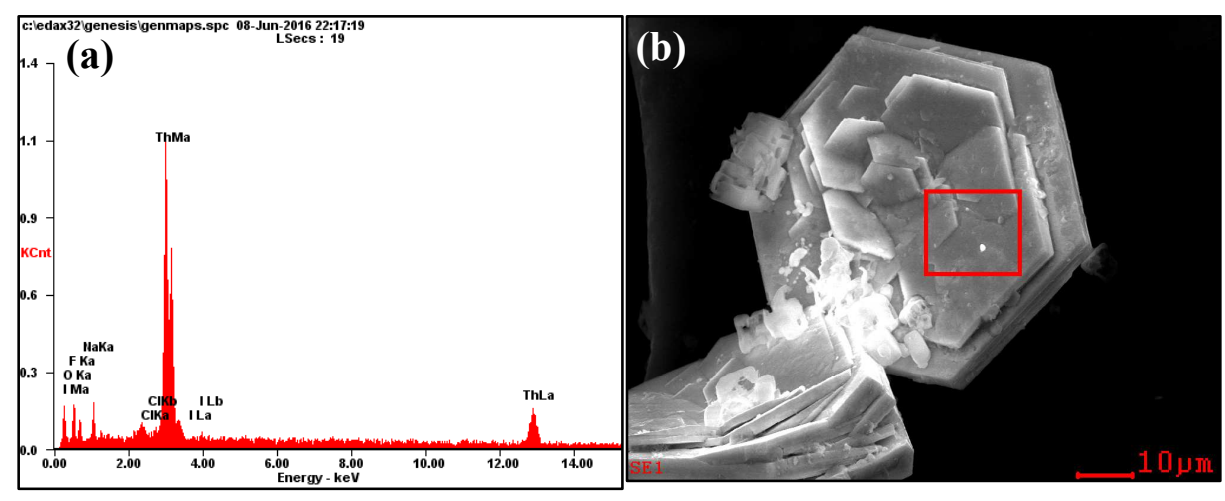


Figure S8. EDS (a) and SEM (b) images of compound ThIOCl soaked in $1 \mathrm{M} \mathrm{NaF}$ solution for $12 \mathrm{~h}$ at room temperature.

\begin{tabular}{|l|l|l|}
\hline & $\boldsymbol{W t} \%$ & $\boldsymbol{A t}^{\boldsymbol{\%}} \boldsymbol{⿴}$ \\
\hline $\boldsymbol{O K}$ & 10.36 & 39.61 \\
\hline $\boldsymbol{F K}$ & 07.00 & 22.54 \\
\hline $\boldsymbol{N a K}$ & 06.16 & 16.39 \\
\hline $\boldsymbol{C l K}$ & 00.57 & 00.98 \\
\hline $\boldsymbol{I L}$ & 02.10 & 01.01 \\
\hline $\boldsymbol{T h L}$ & 73.82 & 19.47 \\
\hline Matrix & Correction & ZAF \\
\hline \hline
\end{tabular}

Table S4. EDS data of compound ThIOCl soaked in $1 \mathrm{M} \mathrm{NaF}$ solutions for $12 \mathrm{~h}$ at room temperature.

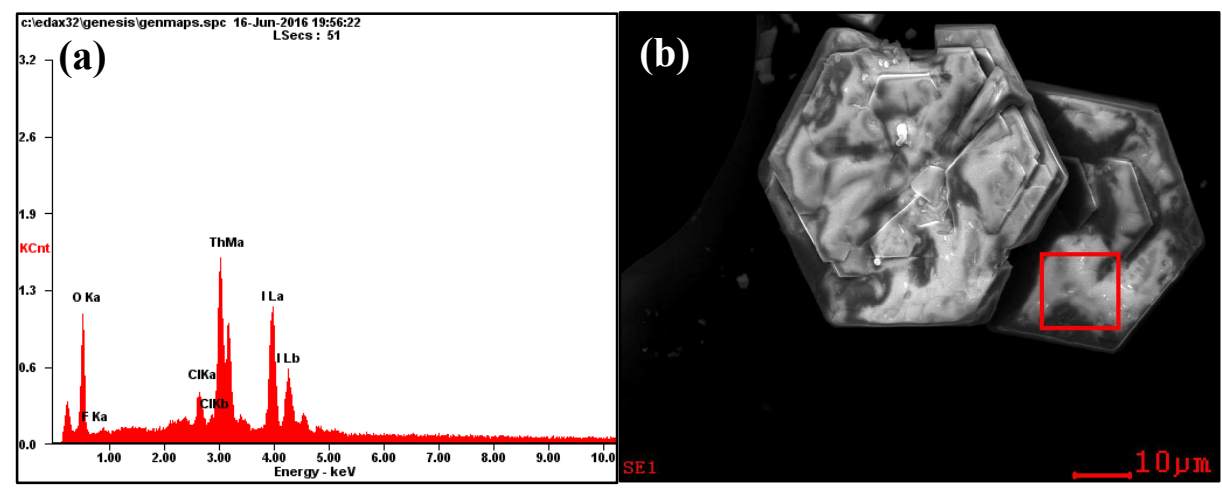

Figure S9. EDS (a) and SEM (b) images of compound ThIOCl soaked in $0.1 \mathrm{M} \mathrm{NaF}$ solutions for $12 \mathrm{~h}$ at room temperature.

\begin{tabular}{|l|l|l|}
\hline Element & Wt\% & At\% \\
\hline OK & 17.29 & 66.18 \\
\hline ClK & 02.46 & 04.25 \\
\hline ThM & 41.86 & 11.05 \\
\hline $\boldsymbol{I L}$ & 38.40 & 18.53 \\
\hline Matrix & Correction & ZAF \\
\hline \hline
\end{tabular}

Table S5. EDS data of compound ThIOCI soaked in $0.1 \mathrm{M} \mathrm{NaF}$ solutions for $12 \mathrm{~h}$ at room temperature. 


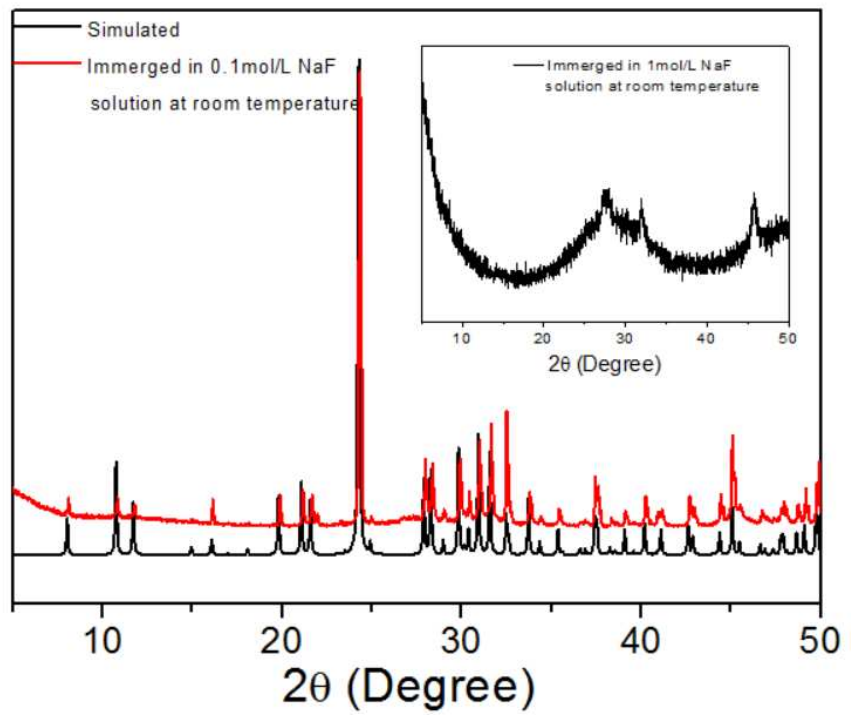

Figure S10. Simulated PXRD patterns and experimental PXRD patterns for ThIOCl immerged in $0.1 \mathrm{M} \mathrm{NaF}$ for $12 \mathrm{~h}$ at room temperature. Inset of Figure S10: experimental PXRD patterns for ThIOCl immerged in $1 \mathrm{M} \mathrm{NaF}$ for $12 \mathrm{~h}$ at room temperature.

\section{S4. Selected bond lengths and angles}

Table S6. Selected bond lengths $(\AA)$ of compound ThIOCl.

\begin{tabular}{llll}
\hline Th1-O1 & $2.379(5)$ & Th1- Th1 & $3.9412(5)$ \\
Th1-O2 & $2.488(6)$ & I1-O2 & $1.820(6)$ \\
Th1-O3 & $2.457(6)$ & I1-O3 & $1.799(6)$ \\
Th1-O4 & $2.422(6)$ & I1-O6 & $1.781(6)$ \\
Th1-O5 & $2.404(6)$ & I2-O4 & $1.801(6)$ \\
Th1-O7 & $2.349(8)$ & I2-O4 & $1.801(6)$ \\
Th1-O7 & $2.412(9)$ & I2-O4 & $1.801(6)$ \\
Th1-O7 & $2.433(9)$ & I3-O5 & $1.786(6)$ \\
Th1- Th1 & $3.9404(4)$ & I3-O5 & $1.786(6)$ \\
Th1- Th1 & $3.9404(5)$ & I3-O5 & $1.786(6)$ \\
\hline
\end{tabular}

Table S7. Selected bond angles $\left(^{\circ}\right)$ of compound ThIOCI.

\begin{tabular}{llllll}
\hline O7-Th1-O1 & $106.3(5)$ & O4-Th1-O7 & $141.8(2)$ & O6-I1-O3 & $98.4(3)$ \\
O7-Th1-O5 & $86.9(3)$ & O7-Th1-O3 & $148.1(3)$ & O6-I1-O2 & $100.9(3)$ \\
O1-Th1-O5 & $137.5(2)$ & O1-Th1-O3 & $77.5(4)$ & O3-I1-O2 & $98.3(3)$ \\
O7-Th1-O7 & $68.8(3)$ & O5-Th1-O3 & $71.5(2)$ & O4-I2-O4 & $99.4(3)$ \\
O1-Th1-O7 & $69.7(3)$ & O7-Th1-O3 & $138.0(3)$ & O4-I2-O4 & $99.4(3)$ \\
\hline
\end{tabular}




\begin{tabular}{llllll}
\hline O5-Th1-O7 & $149.5(3)$ & O4-Th1-O3 & $110.3(2)$ & O4-I2-O4 & $99.4(3)$ \\
O7-Th1-O4 & $84.2(3)$ & O7-Th1-O3 & $84.3(3)$ & O5-I3-O5 & $98.1(3)$ \\
O1-Th1-O4 & $146.87(17)$ & O7-Th1-O2 & $136.3(3)$ & O5-I3-O5 & $98.1(3)$ \\
O5-Th1-O4 & $73.0(2)$ & O1-Th1-O2 & $82.4(3)$ & O5-I3-O5 & $98.1(3)$ \\
O7-Th1-O4 & $86.0(3)$ & O5-Th1-O2 & $115.8(2)$ & Th1-O1-Th1 & $111.8(3)$ \\
O7-Th1-O7 & $68.4(3)$ & O7-Th1-O2 & $75.0(3)$ & Th1-O7-Th1 & $111.8(4)$ \\
O1-Th1-O7 & $69.3(3)$ & O4-Th1-O2 & $69.4(2)$ & Th1-O7-Th1 & $111.0(4)$ \\
O5-Th1-O7 & $79.2(3)$ & O7-Th1-O2 & $148.2(2)$ & Th1-O7-Th1 & $108.8(4)$ \\
O7-Th1-O7 & $106.8(5)$ & O3-Th1-O2 & $75.3(2)$ & & \\
\hline
\end{tabular}

\section{S5. Quantum chemical calculations.}

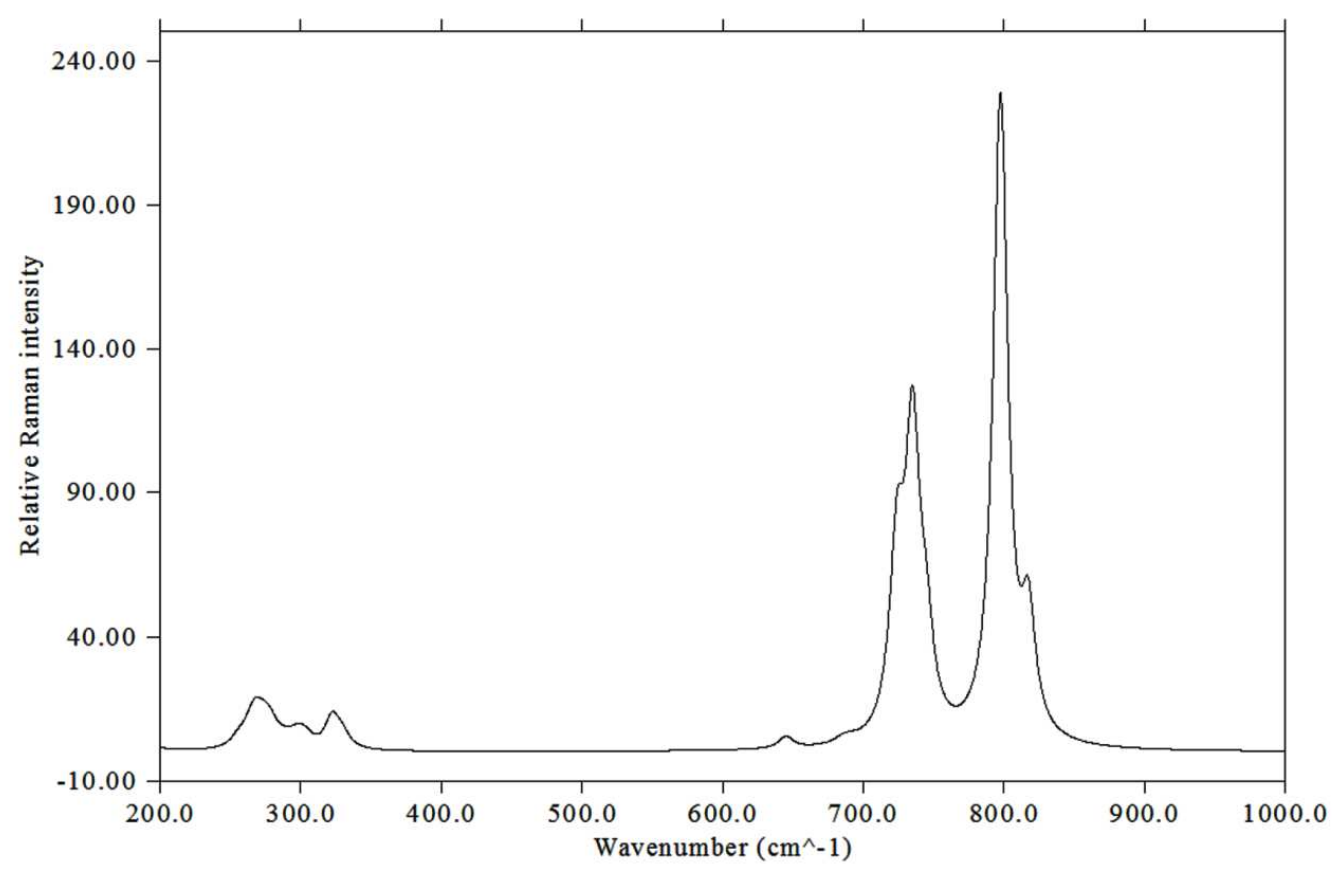

Figure S11. Raman spectra simulation on the model fragment of ThIOCl. 


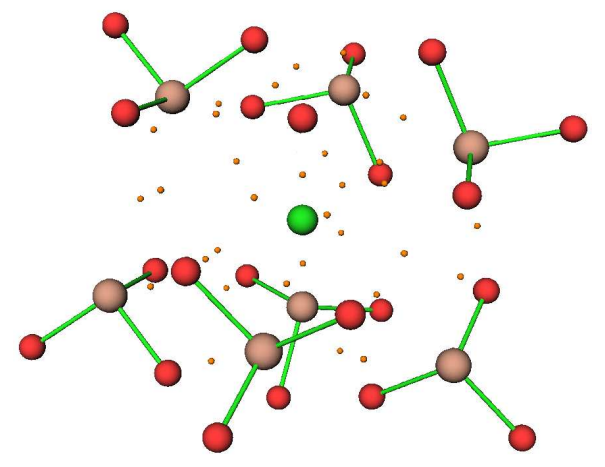

Figure S12. Bond critical points (BCPs, small orange spheres) for the model fragment of ThIOCI (hydrogen atoms are omitted for clarity).

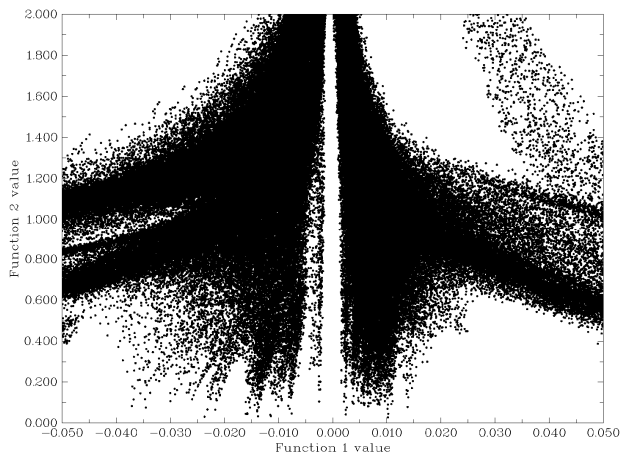

Figure S13. Plots of the reduced density gradient (RDG, Fuction2) versus the electron density multiplied by the sign of the second Hessian eigenvalue $\left(\operatorname{sign}\left(\lambda_{2}\right) \rho\right.$, Fuction1) for the model fragment of ThIOCl (All values are in au).

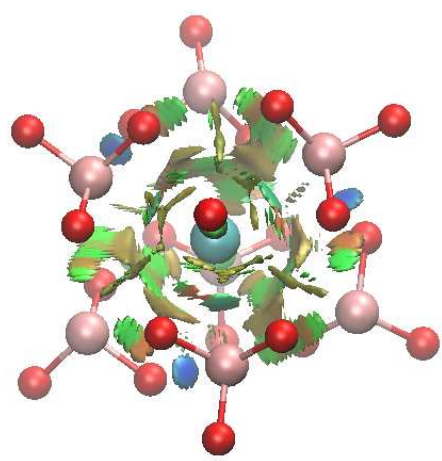


Figure S14. Noncovalent interaction analysis based on the values of $\operatorname{sign}\left(\lambda_{2}\right) \rho$ (blue denotes strong attractive interaction, green indicates van der Waals interaction and red corresponding to strong repulsion) for the model fragment of ThIOCI.

Table S8. Selected interatomic lengths $\left(\mathrm{R}_{\mathrm{ij}}, \AA\right)$ and electron density ( $\rho$, a.u. $)$, laplacian $\left(\nabla^{2} \rho\right.$, a.u. $)$, and energy density (H, a.u.) at the bond critical points for the model fragment of ThIOCI.

\begin{tabular}{lllll}
\hline & $\mathrm{R}_{\mathrm{ij}}$ & $\rho$ & $\nabla^{2} \rho$ & $\mathrm{H}$ \\
\hline & 3.601 & 0.0120 & 0.0266 & 0.0002 \\
& 3.333 & 0.0133 & 0.0478 & 0.0018 \\
$\mathrm{Cl} \cdots \mathrm{I}$ & 3.601 & 0.0120 & 0.0266 & 0.0002 \\
& 3.601 & 0.0120 & 0.0266 & 0.0002 \\
& 3.134 & 0.0233 & 0.0506 & -0.0002 \\
& 3.134 & 0.0233 & 0.0506 & -0.0002 \\
$\mathrm{I} \cdots \mathrm{I}$ & 3.134 & 0.0233 & 0.0506 & -0.0002 \\
& 3.673 & 0.0137 & 0.0292 & -0.0001 \\
$\mathrm{Cl} \cdots \mathrm{O} 1$ & 3.673 & 0.0137 & 0.0292 & -0.0001 \\
\hline & 3.673 & 0.0137 & 0.0292 & -0.0001 \\
& 3.194 & 0.0227 & 0.0812 & 0.0018 \\
\hline
\end{tabular}

